\title{
Modulation of Polyelectrolyte Adsorption on Nanoparticles and Nanochannels by Surface Curvature
}

Facundo Matías Gilles, Fernando M. Boubeta, Omar Azzaroni, Igal Szleifer, and Mario Tagliazucchi

J. Phys. Chem. C, Just Accepted Manuscript • DOI: 10.1021/acs.jpcc.7b12841 • Publication Date (Web): 27 Feb 2018

Downloaded from http://pubs.acs.org on February 27, 2018

\section{Just Accepted}

"Just Accepted" manuscripts have been peer-reviewed and accepted for publication. They are posted online prior to technical editing, formatting for publication and author proofing. The American Chemical Society provides "Just Accepted" as a service to the research community to expedite the dissemination of scientific material as soon as possible after acceptance. "Just Accepted" manuscripts appear in full in PDF format accompanied by an HTML abstract. "Just Accepted" manuscripts have been fully peer reviewed, but should not be considered the official version of record. They are citable by the Digital Object Identifier (DOI®). "Just Accepted" is an optional service offered to authors. Therefore, the "Just Accepted" Web site may not include all articles that will be published in the journal. After a manuscript is technically edited and formatted, it will be removed from the "Just Accepted" Web site and published as an ASAP article. Note that technical editing may introduce minor changes to the manuscript text and/or graphics which could affect content, and all legal disclaimers and ethical guidelines that apply to the journal pertain. ACS cannot be held responsible for errors or consequences arising from the use of information contained in these "Just Accepted" manuscripts. 


\title{
Modulation of Polyelectrolyte Adsorption on
}

\section{Nanoparticles and Nanochannels by Surface}

\section{Curvature}

Facundo M. Gilles ${ }^{1}$, Fernando M. Boubeta ${ }^{3}$, Omar Azzaroni ${ }^{1}$, Igal Szleifer ${ }^{2, *}$ and Mario

Tagliazucchi, $i^{3, *}$

1 Instituto de Investigaciones Fisicoquímicas Teóricas y Aplicadas (INIFTA), CONICET,

Departamento de Química, Facultad de Ciencias Exactas, Universidad Nacional de La Plata, La

Plata 1900, Argentina

2 Department of Biomedical Engineering, Department of Chemistry and Chemistry of Life

Processes Institute, Northwestern University, Evanston, Illinois 60208, United States

3 INQUIMAE-CONICET, Ciudad Universitaria, Pabellón 2, and Ciudad Autónoma de Buenos Aires, Buenos Aires C1428EHA, Argentina

\author{
AUTHOR INFORMATION \\ *Corresponding Authors: \\ Mario Tagliazucchi \\ INQUIMAE-CONICET, Ciudad Universitaria, Pabellón 2, and Ciudad Autónoma de Buenos \\ Aires, Buenos Aires C1428EHA, Argentina. Email: mario@qi.fcen.uba.ar
}




\author{
Igal Szleifer \\ Department of Biomedical Engineering, Department of Chemistry and Chemistry of Life \\ Processes Institute, Northwestern. Email: igalsz@northwestern.edu
}

\begin{abstract}
This paper presents theoretical results on the adsorption of polyelectrolyte chains on surfaces with opposite charge and nanoscale curvature. The theory predicts that increasing surface curvature can either increase or decrease the amount of adsorbed polyelectrolyte, depending on the type of curvature (convex or concave) and whether the polyelectrolyte undercompensates or overcompensates the initial charge of the substrate. For small bulk salt concentration $\left(10^{-4} \mathrm{M}\right)$, increasing the curvature of the surface displaces the adsorption equilibrium of the polyelectrolyte in order to decrease the effective charge density for concave surfaces (nanochannels) or to increase it for convex surfaces (nanoparticles). This behavior is traced back to the dependence the total free energy as a function of the curvature of the surface. For intermediate salt concentrations $(0.01 \mathrm{M}-0.1 \mathrm{M})$, the magnitude of the effect is larger than for low salt concentrations, although the general picture becomes more complex due to the fact that the added salt competes with the polycation to screen the negative charge of the substrate. It is argued that the effect under discussion will be relevant for nanoobjects that have different radii or type of curvature at different locations (i.e. conical nanochannels or cylindrical nanorods with hemispherical tips) as our theory predicts inhomogeneous polyelectrolyte adsorption on their surfaces.
\end{abstract}

1. Introduction 
Polyelectrolyte adsorption on an oppositely charged substrate is a powerful method to modify rough and curved surfaces because the chains of the polyelectrolyte can adapt to the shape of the adsorbing surface. The conformal nature of polyelectrolyte adsorption and the possibility of tailoring film thickness via sequential adsorption of oppositely-charged macromolecules (layer-by-layer self-assembly ${ }^{1}$ ) makes the adsorption of polyelectrolytes on oppositely charged surfaces the first step in many techniques for surface modification of colloids, nanoparticles, nanopores and nanochannels. ${ }^{1-7}$

The interaction of a single polyelectrolyte chain with surfaces of different curvatures have been the subject of recent investigations. ${ }^{8-13}$ The adsorption of polyelectrolytes from solution to form a film on planar and convex surfaces has been also extensively studied in literature and it is relatively well understood. ${ }^{14-22}$ However, the adsorption of polyelectrolytes from solution on concave surfaces is still poorly investigated. Moreover, the effects of the radius and type of surface curvature (concave vs convex) on the adsorption of polyelectrolytes remain largely unexplored. Understanding polyelectrolyte adsorption on curved surfaces is particularly important in those systems where different radii of curvature or types of curvature coexists, for example nanorods ${ }^{3}$ (which have cylindrical bodies and hemispherical tips) and conical nanochannels (which have narrow nanometric tips and broad micrometric bases). ${ }^{2}$ The importance of surface curvature for polyelectrolyte adsorption is anticipated from the fact that surface curvature determines the volume element at a given distance from the surface, $d$, which modulates the electrostatic and non-electrostatic interactions among polyelectrolytes. ${ }^{23}$ The volume element at a distance $d$ from the surface is constant for planar surfaces, it increases as $\sim 1$ $+d / R$ for cylinders (where $R$ is the radius of the cylinder), decreases as $\sim 1-d / R$ for cylindrical channels and increases as $\sim(1+d / R)^{2}$ for spherical particles. This discussion stresses the importance of competing lengthscales in polyelectrolyte adsorption on curved nanoobjects as radii of curvature of a few tens of nanometers are commensurable with two important lengthscales: the dimension of the polyelectrolyte chain and the Debye length of the solution.

In this work, we present the results of our systematic theoretical investigation of the effect of surface curvature on polyelectrolyte adsorption. We show that increasing the curvature of the channel may increase or decrease the amount of adsorbed polyelectrolyte, depending on the concentration of the polyelectrolyte and salt ions in solution and the type of curvature 
(convex or concave). Finally, we address the potential experimental relevance of our findings by systematically comparing the amount of adsorbed polyelectrolyte on surfaces of equal charge but opposite curvature.

\section{Theoretical Methods}

The system under study consists of a charged surface in equilibrium with a solution of an oppositely charged polyelectrolyte. We will assume that the chargeable groups in the polyelectrolyte and the surface are fully dissociated (i.e. they are strong electrolytes). The present study comprises a polycation interacting with a negatively charged surface, but, of course, our results can be straightforwardly applied to the case of a polyanion interacting with a positive surface. We theoretically study polyelectrolyte adsorption on curved surfaces with a previously developed molecular theory, ${ }^{4,23-26}$ which explicitly considers the shape, size, charge, and conformations of all molecular species in the system. This approach has been used in the past to study different systems in soft-matter science and its predictions have been found to be in good agreement with experimental observations. ${ }^{4-5,27}$ This theoretical approach consists in writing down an approximation for the proper thermodynamic potential of the system, which in the present case is the grand potential $\Omega$,

$$
\begin{aligned}
& \beta \Omega=\int G(r) \rho_{\text {chain }}(r)\left[\ln \left(\rho_{\text {chain }}(r) v_{w}\right)-1+\beta \mu_{\text {chain }}^{o}\right] d r+\sum_{i=c, a, w} \int G(r) \rho_{i}(r)\left[\ln \left(\rho_{i}(r) v_{w}\right)-1+\beta \mu_{i}^{o}\right] d r+ \\
& \int G(r) \rho_{\text {chain }}(r) \sum_{\alpha} P(r, \alpha) \ln (P(r, \alpha)) d r+\int G(r)\left[\left\langle\rho_{Q}(r)\right\rangle \beta \psi(r)-\frac{1}{2} \beta \varepsilon\left(\nabla_{r} \psi(r)\right)^{2}\right] d r+G(R) \sigma_{\text {surf }} \beta \psi(R) \\
& -\sum_{i=a, c, \text { chain }, w} \beta \mu_{i} \int G(r) \rho_{i}(r) d r
\end{aligned}
$$

where $\beta$ is the inverse temperature, i.e. $1 / k_{\mathrm{b}} \mathrm{T}$.

The grand potential $\Omega$ is a functional of the position-dependent densities of the mobile species, $\rho_{\mathrm{i}}(r)$ (with $\mathrm{i}=c, a, w$ and chain for cations, anions, water molecules and polyelectrolyte chains, respectively), the probability distribution function of the polyelectrolyte conformations, $P(r, \alpha)$ and the position-dependent electrostatic potential, $\psi(r)$. The first term in equation (1) corresponds to the mixing entropy of free polymer chains plus the contribution from their standard chemical potential $\left(\beta \mu_{\text {chain }}^{0}\right)$. In this term, $\rho_{\text {chain }}(r)$ is the number density of chains at $r$ 
(we use the convention that assigns the position of a chain by the position of the segment closest to the surface) and $v_{\mathrm{w}}$ is the volume a water molecule. Note that this volume appears in the translational entropy of polyelectrolyte chains because we use $v_{\mathrm{w}}$ as the reference volume in our theory (the choice of the reference volume has no thermodynamic or structural consequences since choosing a different reference volume will just shift the value of $\beta \mu_{\text {chain }}^{0}$ ). The function $\mathrm{G}(r)$ defines the geometry of the calculation, i.e. it determines the volume element as a function of the distance from the origin, as we explain below. The second term in eq (1) is the contribution from the translational entropies of small molecules in solution (cations, anions and water molecules). The third term is the contribution from the conformational entropy of polyelectrolyte chains; in this term, $P(r, \alpha)$ is the probability of having a chain in the conformation $\alpha$ at position $r$, where $\alpha$ labels the conformation of the polymer chain. The fourth and fifth terms are the electrostatic energy of the system, where $\varepsilon$ is the dielectric constant and $\left\langle\rho_{\mathrm{Q}}(r)\right\rangle$ is the density of charges at $r$, which results from adding the charge densities at $r$ of cations, anions and polyelectrolyte chains. In the fifth term, $\sigma_{\text {surf }}$ is the surface charge density of the substrate. The last term in eq. (1) is equal to the sum of $-\mu_{\mathrm{i}} N_{\mathrm{i}}$ for all species in the system, where $\mu_{\mathrm{i}}$ is the chemical potential of species $\mathrm{i}$ and $N_{\mathrm{i}}$ is the total number of particles of species $\mathrm{i}$, which is calculated in eq. (1) by integrating $\rho_{\mathrm{i}}(r)$ over the entire system. The terms $-\mu_{\mathrm{i}} N_{\mathrm{i}}$ are included in $\Omega$ because we describe a system where the chemical potentials of all species are fixed by their bulk concentrations (i.e. $\Omega$ is a grand potential). Note that we do not include nonelectrostatic polyelectrolyte-surface or polyelectrolyte-polyelectrolyte attractions (the polyelectrolyte is in a good solvent) in eq. (1).

Our theory takes advantage of the symmetry of the system by assuming that there are inhomogeneities only in the direction normal to the planar, cylindrical or spherical substrate. Therefore, the position within the system in all functions in eq. (1) is given by the position in the normal direction, $r$. We use $r=0$ for the center of the spherical nanoparticle, the central axis of the cylindrical nanochannel or nanorod or the position of the planar surface. In the case of nanoparticles, nanorods or nanochannels, their surface is located at $r=R$. The function $\mathrm{G}(r)$ is given by the type of geometry (planar, cylindrical or spherical): $\mathrm{G}(r) \mathrm{d} r$ corresponds to the volume element as a function of the distance from the origin. More specifically, $\mathrm{G}(r) \mathrm{d} r=A \mathrm{~d} r$ for a planar surface (where $A$ is the area of the surface), $\mathrm{G}(r) \mathrm{d} r=2 \pi L r \mathrm{~d} r$ for cylindrical nanorods or 
nanochannels (where $L$ is the length of the cylinder) and $\mathrm{G}(r) \mathrm{d} r=4 \pi r^{2} \mathrm{~d} r$ for spherical particles. The approximation of inhomogeneities only in the normal direction implies that we describe very long (length much larger than radius) cylindrical rods and channels, so that end effects are negligible.

The grand potential $\Omega$ does not contain an explicit term accounting for steric repulsions. Intramolecular steric repulsions for the polyelectrolyte chains are accounted for by considering only self-avoiding conformations. All intermolecular steric repulsions are included at a meanfield level through the use of a packing constraint:

$$
\left\langle\rho_{p}(r)\right\rangle v_{p}+\sum_{i=a, c, w} \rho_{i}(r) v_{i}=1 \quad(\text { for all } r)
$$

where $v_{\mathrm{i}}$ is the volume of species $\mathrm{i}(\mathrm{i}=a, c, w$ or $p$ for anions, cations, water molecules and polyelectrolyte segments). In this equation, $\left\langle\rho_{\mathrm{p}}(r)\right\rangle$ is the density of polymer segments at $r$, which is not an independent thermodynamic variable since it can be calculated from $\rho_{\text {chain }}(r)$ and $P(r, \alpha)$ :

$$
\left\langle\rho_{p}(r)\right\rangle=\int \frac{G\left(r^{\prime}\right)}{G(r)} \rho_{\text {chain }}\left(r^{\prime}\right) \sum_{\alpha} P\left(r^{\prime}, \alpha\right) n\left(r^{\prime}, \alpha, r\right) d r^{\prime}
$$

where $n\left(r^{\prime}, \alpha, r\right) \mathrm{d} r$ is the number of segments that a polyelectrolyte chain in conformation $\alpha$ and with its segment closest to the surface at $r$ ' has in the region located between $r$ and $r+\mathrm{d} r$. Note that this function depends on the geometry of the system, i.e. for a given conformation $\alpha$ and position of the segment closest to the surface at $r^{\prime}, n\left(r^{\prime}, \alpha, r\right)$ is different in planar, cylindrical or spherical geometries.

We determine the equilibrium state of the system by finding the stationary point of the grand potential subjected to three constrains that are enforced using Lagrange multipliers. The first constraint is the packing constraint (eq. (2)). The second constraint is the normalization of $P(r$, $\alpha)$,

$$
\sum_{\alpha} P(\alpha, r)=1 \quad(\text { for all } r)
$$


Finally, the third constraint is global electroneutrality:

$$
\int G(r)\left\langle\rho_{Q}(r)\right\rangle d r=0
$$

Considering the constraints, we look for the stationary point of the following potential:

$$
\begin{aligned}
& \beta W=\beta \Omega-\int \beta \pi(r) G(r)\left[\left\langle\rho_{p}(r)\right\rangle v_{p}+\sum_{i=a, c, w} \rho_{i}(r) v_{i}-1\right] d r \\
& -\lambda \int G(r)\left\langle\rho_{q}(r)\right\rangle d r-\int G(r) \xi(r)\left(\sum_{\alpha} P(r, \alpha)-1\right) d r
\end{aligned}
$$

In this expression, the global electroneutrality condition is enforced by the Lagrange multiplier $\lambda$, the normalization is enforced by the Lagrange multiplier $\xi(r) \mathrm{G}(r)$ and the packing constraint at $r$ by the Lagrange multiplier $\beta \pi(r) \mathrm{G}(r)$. We now find the extremum of the grand potential with respect to $\rho_{\mathrm{i}}(r), P(r, \alpha)$ and $\psi(r)$.

The extremum of the grand potential in eq. (3) with respect to the electrostatic potential leads to the Poisson equation:

$$
\nabla^{2} \psi(r)=-\frac{1}{\varepsilon}\left\langle\rho_{Q}(r)\right\rangle
$$

We have not included in this equation the Lagrange multiplier associated with global electroneutrality, $\lambda$, because $\lambda$ appears as an additive constant to $\psi$ and therefore has no thermodynamic consequences due to the arbitrary choice of the zero for the electrostatic potential. Note that the Laplacian operator is $\nabla^{2}=\partial^{2} / \partial r^{2}$ for planar geometry, $\nabla^{2}=\partial^{2} / \partial r^{2}+(1 / r) \partial / \partial r$ for cylindrical geometry or $\nabla^{2}=\partial^{2} / \partial r^{2}+(2 / r) \partial / \partial r$ for spherical geometry. The boundary condition of the electrostatic potential at the surface is:

$$
\left.\nabla_{r} \psi(r)\right|_{r=R} \hat{\mathbf{n}}=-\frac{\sigma_{\text {surf }}}{\varepsilon}
$$


where $\hat{\mathbf{n}}$ is a vector normal to the surface and $\sigma_{\text {surf }}$ is the charge of the surface. The boundary condition at the bulk solution is $\psi^{\text {bulk }}=0$. In the case of the cylindrical channel, we should also consider the symmetry condition at the center of the channel, $r=0$ :

$$
\left.\nabla_{r} \psi(r)\right|_{r=0}=0
$$

The stationary point of the potential $\beta W$ with respect to $P(r, \alpha)$ results in:

$$
P(r, \alpha)=\frac{1}{q(r)} \exp \left[-\int n\left(r, \alpha, r^{\prime}\right)\left(\beta \psi\left(r^{\prime}\right) q_{p}+\beta \pi\left(r^{\prime}\right) v_{p}\right) d r^{\prime}\right]
$$

In this expression, $q(r)$ is a single-chain partition function for the chains that have their segment closest to the surface at $r$. This partition function ensures the normalization condition, eq (4) and it is related to the Langrage multiplier $\xi(r), q(r)=\exp \left(\xi(r) / \rho_{\text {chain }}(r)+1\right)$. It is interesting to note that $P(r, \alpha)$ depends on the electrostatic potential at different positions in the system. On the other hand, the electrostatic potential profile depends on the charge density $\left\langle\rho_{\mathrm{Q}}(r)\right\rangle$, which contains contributions from the polyelectrolytes (as well as the mobile ions). While our theory treats electrostatic interactions at a mean field level, the non-local nature of the coupling between polyelectrolyte conformations and the electrostatic potential profile introduces correlations into the theory which are important to properly describe some aspects of polyelectrolyte adsorption, as we show in the results section.

The extremum of $\beta W$ with respect to $\rho_{\mathrm{i}}(r)$ for $i=a$ and $c$ results in:

$\rho_{i}(r)=v_{w}^{-1} \exp \left(\beta \mu_{\mathrm{i}}-\beta \mu_{i}^{0}-\beta v_{\mathrm{i}} \pi(\mathrm{r})-\beta q_{\mathrm{i}} \psi(\mathrm{r})\right)$

and the extremum with respect to $\rho_{\mathrm{w}}(r)$ yields,

$\rho_{w}(r)=v_{w}^{-1} \exp \left(\beta \mu_{w}-\beta \mu_{w}^{0}-\beta v_{w} \pi(\mathrm{r})\right)$ 
These expressions show that $\pi(r)$, the Lagrange multiplier associated with the packing constraint, plays the role of a local osmotic pressure.

The extremum with respect to $\rho_{\text {chains }}(r)$ gives after some rearrangements and substitutions,

$$
\rho_{\text {chains }}(r)=q(r) v_{w}^{-1} \exp \left(\beta \mu_{\text {chain }}-\beta \mu_{\text {chains }}^{0}\right)
$$

The chemical potentials of water, ions and polyelectrolyte chains required in eqs (11)-(13) are obtained from the composition of the bulk as:

$\left(\beta \mu_{\mathrm{i}}-\beta \mu_{i}^{0}\right)=\ln \left(\rho_{i}^{b u l k} v_{w}\right)+\beta v_{\mathrm{i}} \pi^{b u l k}$

for anions and cations,

$$
\left(\beta \mu_{w}-\beta \mu_{w}^{0}\right)=\ln \left(\rho_{w}^{b u l k} v_{w}\right)+\beta v_{w} \pi^{b u l k}
$$

for water molecules and,

$$
\left(\beta \mu_{\text {chain }}-\beta \mu_{\text {chains }}^{0}\right)=\ln \left(\rho_{\text {chains }}^{\text {bulk }} v_{w}\right)-\ln q^{\text {bulk }}
$$

for polyelectrolyte chains.

In practice, due to the presence of the packing constraint, it is also possible to establish a relationship between the chemical potential of the different species in solution and rewrite eqs (11)-(16) in terms of exchange chemical potentials. ${ }^{24}$

We solve the set of integro-differential equations (2)-(4) and (7)-(13) by discretizing the equations and solving the discretized equations with numerical methods (see Supporting Information). As an outcome of the theory, we obtain equilibrium structural (including the 
functions $\rho_{\mathrm{i}}(r), P(r, \alpha)$ and $\left.\psi(r)\right)$ and thermodynamic (including the grand potential, $\Omega$ ) properties.

In order to present the results of our calculations, we will define two useful variables. First, we define $\sigma_{p o l}$ as the number density of charges of the adsorbed polyelectrolytes. In our model, each polyelectrolyte has a chain length of $N$ and each segment has a positive charge, thus $\sigma_{p o l}$ is equal to the product of the surface density of adsorbed polyelectrolyte chains by the chain length. We consider a chain to be adsorbed if it has at least one segment located at distance equal to or small than $\delta$ from the surface (we use $\delta$ equal to the segment length, $0.5 \mathrm{~nm}$, in all calculations). Based on this definition, $\sigma_{p o l}$ is given by:

$$
\sigma_{\text {pol }}=q_{p} N \int_{R}^{R+\delta}\left(\frac{G(r)}{G(R)}\right) \rho_{\text {chain }}(r) d r
$$

We also define the effective surface charge density, $\sigma_{\text {eff }}$, as the sum of the charge density due to polyelectrolyte chains attached to the surface $\left(\sigma_{p o l}\right)$ and the charge density of the negatively charged surface $\left(\sigma_{\text {surf }}\right)$, i.e. $\sigma_{\text {eff }}=\sigma_{\text {pol }}+\sigma_{\text {surf }}$ (note that $\sigma_{\text {pol }}$ is positive and $\sigma_{\text {surf }}$ is negative). Note that in this definition, all segments of adsorbed polyelectrolyte chains contribute to the effective charge density of the surface.

\section{Results}

\subsection{Polyelectrolyte adsorption on nanochannels}

We start our analysis by considering polyelectrolyte adsorption on a (very long) cylindrical nanochannel. Figure 1 shows our predictions for $\sigma_{p o l}$ (number density of charges of the adsorbed polyelectrolytes, left axis) and $\sigma_{e f f}$ (effective surface charge density, right axis) and as a function of channel radius for different concentrations of polyelectrolyte and added salt in solution. 

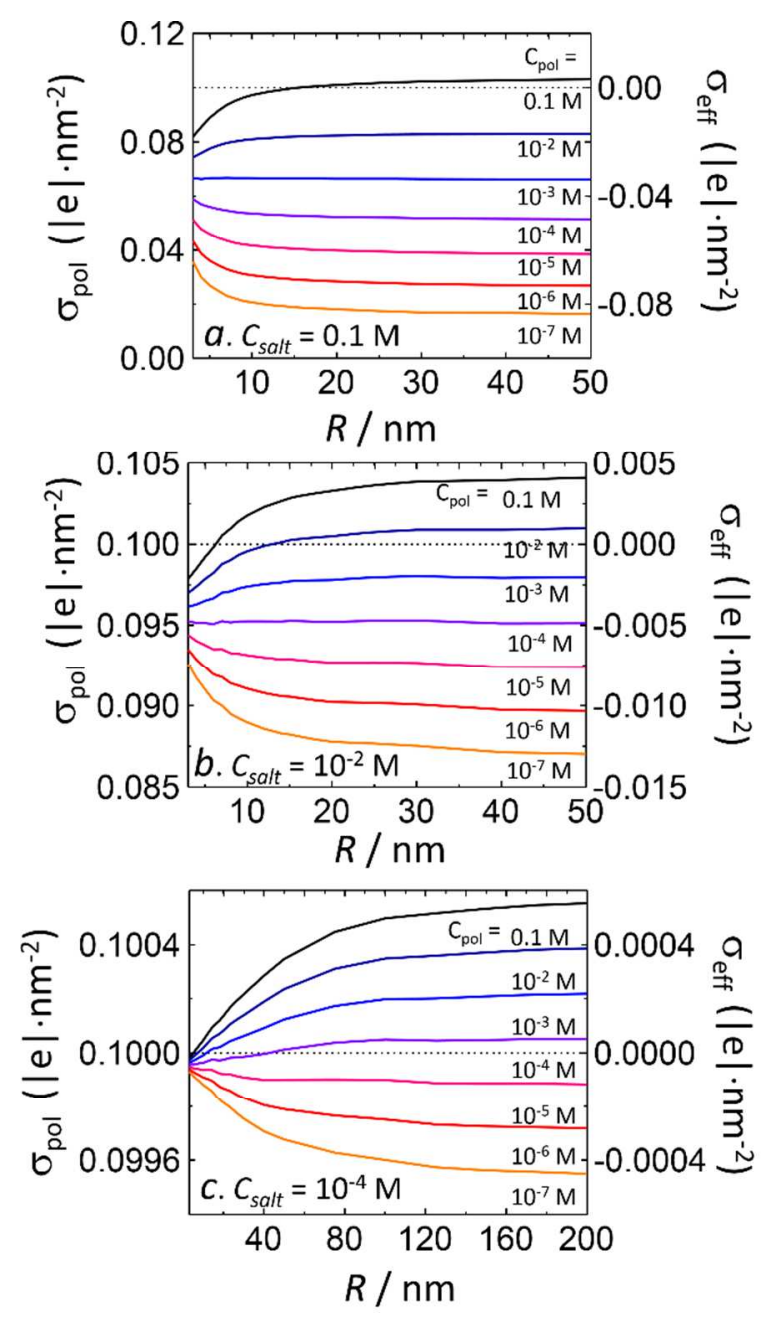

Figure 1. Charge density of positively charged polyelectrolytes adsorbed on the surface of a negatively charged nanochannel, $\sigma_{\text {pol }}$ (left axis in the plot), and effective net charge density of the surface, $\sigma_{e f f}=\sigma_{p o l}+\sigma_{\text {surf, }}$ (right axis) as a function of radius of the nanochannel. Different curves indicate different bulk concentrations of polyelectrolyte, $C_{\mathrm{pol}}$ (expressed in terms of the molar concentration of monomers), as indicated below each curve. The bulk salt concentration was $C_{\text {salt }}=0.1 \mathrm{M}(\mathrm{a}), 0.01 \mathrm{M}(\mathrm{b})$ or $10^{-4} \mathrm{M}(\mathrm{b})$. Other calculation parameters: chain length, $N=50$; charge density of the surface, $\sigma_{\text {surf }}=-0.1|\mathrm{e}| \cdot \mathrm{nm}^{-2}$.

Strikingly, the theory predicts that the effective surface charge could either increase or decrease when increasing the radius of the nanochannel. Moreover, in some conditions (for 
example $C_{p o l}=10^{-2} \mathrm{M}$ in Figure $1 \mathrm{~b}$ ), the effective surface charge density, $\sigma_{\text {eff, }}$ can change its sign as the radius of the channel decreases, going from a charge overcompensation scenario $\left(\sigma_{\text {eff }}>0\right.$, that is, polyelectrolyte charge density larger than surface charge density) to charge undercompensation ( $\sigma_{\text {eff }}<0$, polyelectrolyte charge density smaller than surface charge density). The effect of curvature on the amount of adsorbed polyelectrolyte shown in Figure $1 \mathrm{a}\left(C_{\text {salt }}=0.1\right.$ $\mathrm{M})$ and Figure $1 \mathrm{~b}\left(C_{\text {salt }}=0.01 \mathrm{M}\right)$ is relatively small, yielding a maximum change of adsorption of around 25\% when going from the highest $(3 \mathrm{~nm})$ to the lowest $(50 \mathrm{~nm})$ curvatures considered in the plots. At the end of this communication, we analyze in detail the conditions that maximize the effect of curvature on the amount of adsorbed polyelectrolyte and discuss its potential experimental relevance.

In order to provide physical insights on the results in Figures 1a and 1b, let us analyze the case of very low ionic strengths $\left(C_{\text {salt }}=10^{-4} \mathrm{M}\right.$, shown in Figure 1c). In this case, the concentration of salt cations is very small and thus the charges on the surface must be compensated almost quantitatively by those of the adsorbed polyelectrolyte. Note that the scale of the surface charge density in Figure 1c indicates that the effect of channel radius on adsorption is very small and that the deviations from stoichiometric charge compensation $\left(\sigma_{\text {eff }}=0\right)$ are always smaller than $0.5 \%$. Despite the very small magnitude of the effect for $C_{\text {salt }}=10^{-4} \mathrm{M}$, the results in Figure 1c show a very interesting relationship between the amount of adsorbed polyelectrolyte, the type of charge compensation and the radius of the channel. When the nanochannel charge is overcompensated by the polyelectrolyte $\left(\sigma_{\text {eff }}>0\right.$, which occurs for large bulk polyelectrolyte concentrations, see Figure 1c), decreasing the diameter of the channel leads to a decrease in the amount of adsorbed polyelectrolyte. On the other hand, when the charge of the wall is undercompensated by the polyelectrolyte $\left(\sigma_{\text {eff }}<0\right.$, which occurs for small polyelectrolyte bulk concentrations), then the amount of adsorbed polyelectrolyte increases for decreasing diameter. Finally, for $\sigma_{\text {eff }} \sim 0$, the amount of adsorbed polyelectrolyte is almost constant with the diameter of the channel (which occurs for $C_{\mathrm{pol}}$ between $10^{-3}$ and $10^{-4} \mathrm{M}$ ).

We explain the results in Figure 1c as follows. In the case of a charged surface in contact with a salt solution only (without polyelectrolyte chains in solution), decreasing the radius of the channel for a constant surface charge, leads to an increase in the grand potential of the system, $\Omega$ (see Figure 2a). Such increase in $\Omega$ is caused by the fact that narrowing the channel confines the 
cation counterions in the electrical double layer. The confinement of counterions is evidenced in Figure $2 b$, which shows that the concentration of cations in the double layer is larger for the cylindrical channel than for the flat surface. In the presence of the polyelectrolyte in solution, the increase of the grand potential with increasing curvature can be alleviated by decreasing the effective surface charge, $\sigma_{\text {eff. }}$ The decrease of $\sigma_{\text {eff }}$ can be achieved by adsorbing polyelectrolytes from solution if $\sigma_{\text {eff }}<0$, or by desorbing them from the surface if $\sigma_{\text {eff }}>0$, which explains the results in Figure 1c.

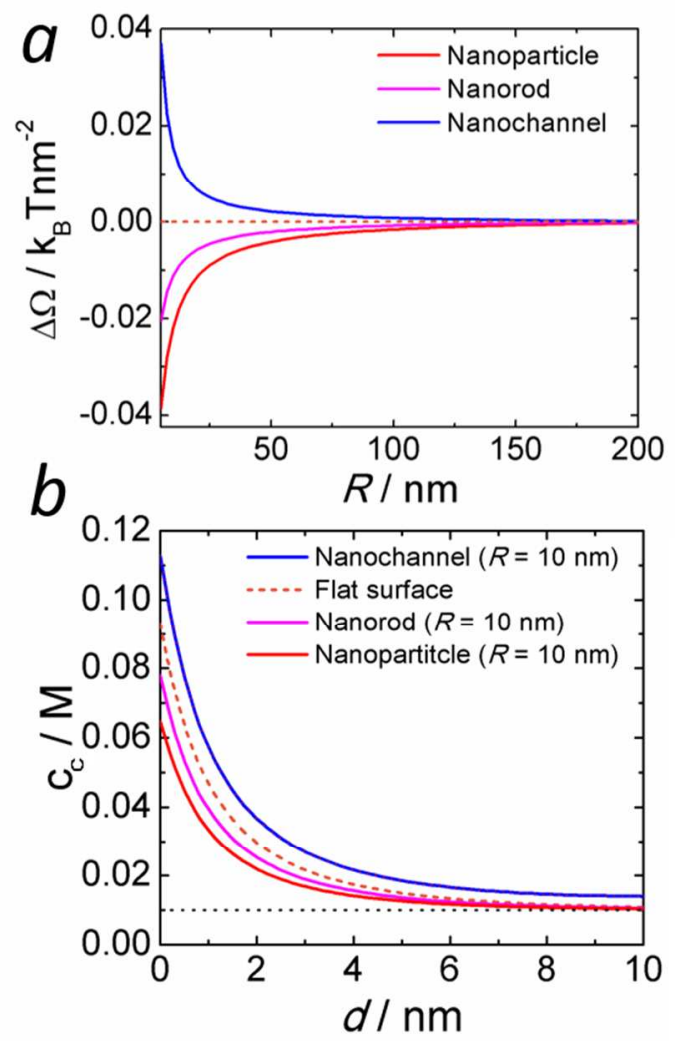

Figure 2. a. Difference of grand potential between a curved surface (nanochannel, nanorod or spherical nanoparticle) and a planar surface as a function of the radius of the curved surface. Calculation conditions: $\sigma_{\text {surf }}=-0.1|\mathrm{e}| \cdot \mathrm{nm}^{-2}, C_{\text {salt }}=0.01 \mathrm{M}$. b. Concentration of cations as a function of the distance from the surface for the systems in panel a (the radius of curvature in this panel is $R=10 \mathrm{~nm}$ ). The cation concentration in bulk is shown with dotted lines. 
The example in Fig. 1c is useful to analyze the mechanism by which nanoconfinement regulates the amount of adsorbed polyelectrolyte, but, as we mentioned above, the magnitude of the effect in that case is very small. The effect is small in this case because, in the absence of salt ions, the only mechanism of charge compensation is polyelectrolyte adsorption; therefore, the amount of adsorbed polyelectrolyte cannot deviate too much from that required for stoichiometric charge compensation (i.e. $\sigma_{\text {eff }}=0$ ) without incurring in large energetic penalties. On the other hand, for intermediate ionic strengths (e.g. $C_{\text {salt }}=0.1 \mathrm{M}$ and $0.01 \mathrm{M}$ for Figures $1 \mathrm{a}$ and $1 b$, respectively), the cations of the salt compete with the polycation to compensate the negative charges of the substrate, which allows for significant deviations from stoichiometric charge compensation and thus enhances the effect of nanoconfinement on polyelectrolyte adsorption.

The competition between the polycation and the small salt cations to compensate the negative charges on the surface is apparent Figure 3, which shows the molar concentration of salt ions and polyelectrolyte segments as a function of the distance to the center of the channel for $C_{\text {salt }}=0.1 \mathrm{M}$ (panel a) and $C_{\text {salt }}=10^{-4} \mathrm{M}$ (panel b). In the case of $C_{\text {salt }}=0.1 \mathrm{M}$, there is an increase in the concentration of cations near the surface, which indicates that salts cations compete with the polyelectrolyte to screen the negative charges of the substrate. Note also the small depletion of cations and a small enhancement of anion concentration around $12 \mathrm{~nm}$ in panel a. This effect is due to the inversion of the electrostatic potential at the top of the polyelectrolyte layer (this inversion does not imply overcompensation of the net charge of the surface. i.e. $\sigma_{\mathrm{eff}}<0$ for these conditions). In the case of $C_{\text {salt }}=10^{-4} \mathrm{M}$, the concentration of cations near the surface is negligible compared to that of the polyelectrolyte. The competition between polyelectrolyte chains and small ions to compensate the charge of the surface in Figure 3a allows deviations from perfect polyelectrolyte-surface stoichiometric compensation. It is also responsible for the fact that the effective surface charge for which the amount of adsorbed polyelectrolyte is independent on surface curvature occurs for $\sigma_{\text {eff }} \sim 0$ for $C_{\text {salt }}=10^{-4} \mathrm{M}$ (Figure $1 \mathrm{c})$, but for $\sigma_{\mathrm{eff}}<0$ for higher ionic strengths. 


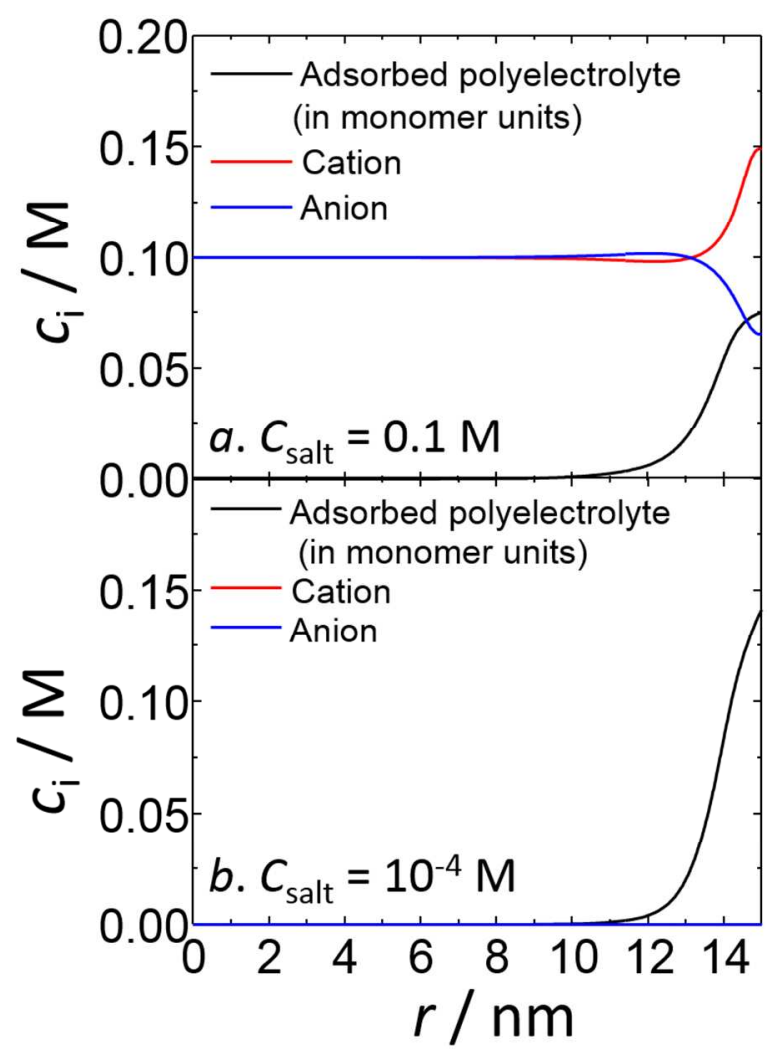

Figure 3. Molar concentration profiles of the adsorbed polyelectrolyte, cations and anions as a function of the distance from the center of a channel of radius $R=15 \mathrm{~nm}(r=15 \mathrm{~nm}$ corresponds to the surface of the channel). Calculation conditions: chain length, $N=50$; bulk salt concentration, $C_{\text {salt }}=0.1 \mathrm{M}$ (panel a) or $10^{-4} \mathrm{M}$ (panel b); polymer concentration in the bulk, $C_{\text {pol }}$ $=10^{-3} \mathrm{M}$, channel radius, $R=15 \mathrm{~nm}$; substrate surface charge, $\sigma_{\text {surf }}=-0.1|\mathrm{e}| \cdot \mathrm{nm}^{-2}$. Note that the concentration of polyelectrolyte is expressed as molar concentration of monomer units.

\subsection{Polyelectrolyte adsorption on nanorods and nanoparticles}

So far we have analyzed the effect of channel diameter on polyelectrolyte adsorption for cylindrical nanochannels. An important question is whether the mechanisms discussed above also hold for convex surfaces, namely, nanoparticles and nanorods. Figure $2 b$ shows that the concentration of cations in the double layer is smaller for a spherical nanoparticle than for a cylindrical nanorod, which in turn is smaller than that for the planar surface. Therefore, increasing the curvature of a convex charged surface decreases the degree of confinement of the counterions in the double layer and decreases the grand potential of the system (Figure 2a). Hence, one expects that the effect of curvature for polyelectrolyte adsorption on convex surfaces 
will be the opposite to that described above for nanochannels: we expect a decrease of polyelectrolyte adsorption with increasing curvature if $\sigma_{\text {eff }}<0$ or and an increase if $\sigma_{\text {eff }}>0$ (i.e. the system responds to increasing curvature by changing the amount of adsorbed polyelectrolyte in order to increase the absolute value of $\left.\sigma_{\text {eff }}\right)$. This argument is in full agreement with the predictions of the theory, see Figure 4 for long cylindrical nanorods and spherical nanoparticles. Interestingly, Figure 4 shows that for a given polyelectrolyte and salt concentrations, the effect of curvature radii on polyelectrolyte adsorption is stronger for the spherical nanoparticle than for the cylindrical rod, which is due to the fact that the volume element at a given distance from the surface increases as $\sim 1+d / R$ for a cylinder and as $\sim(1+d / R)^{2}$ for a sphere. 
Cylindrical Nanorod
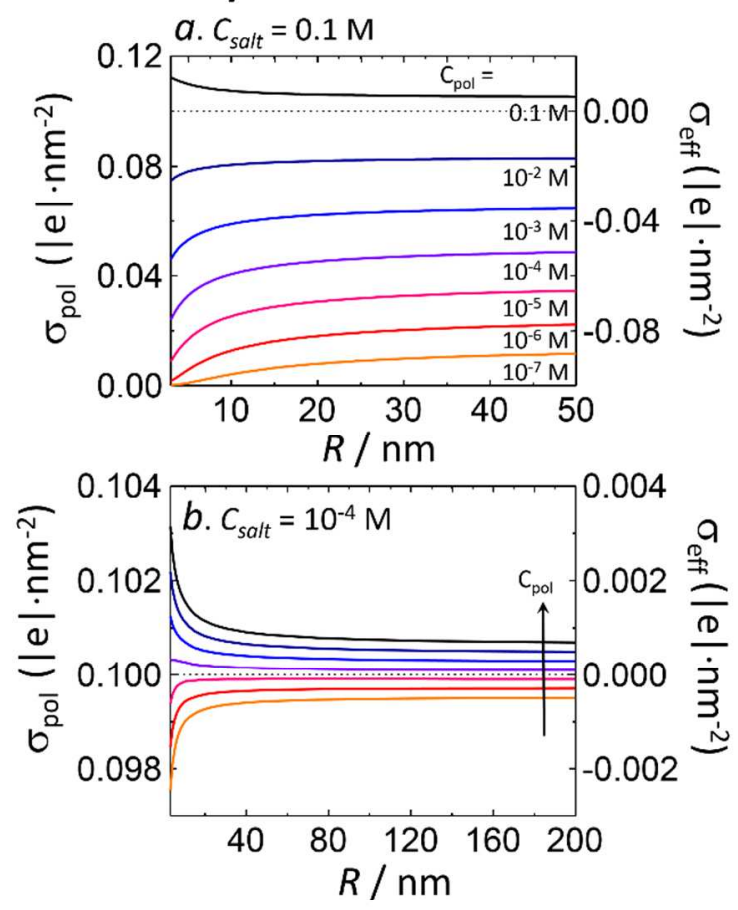

Spherical Nanoparticle
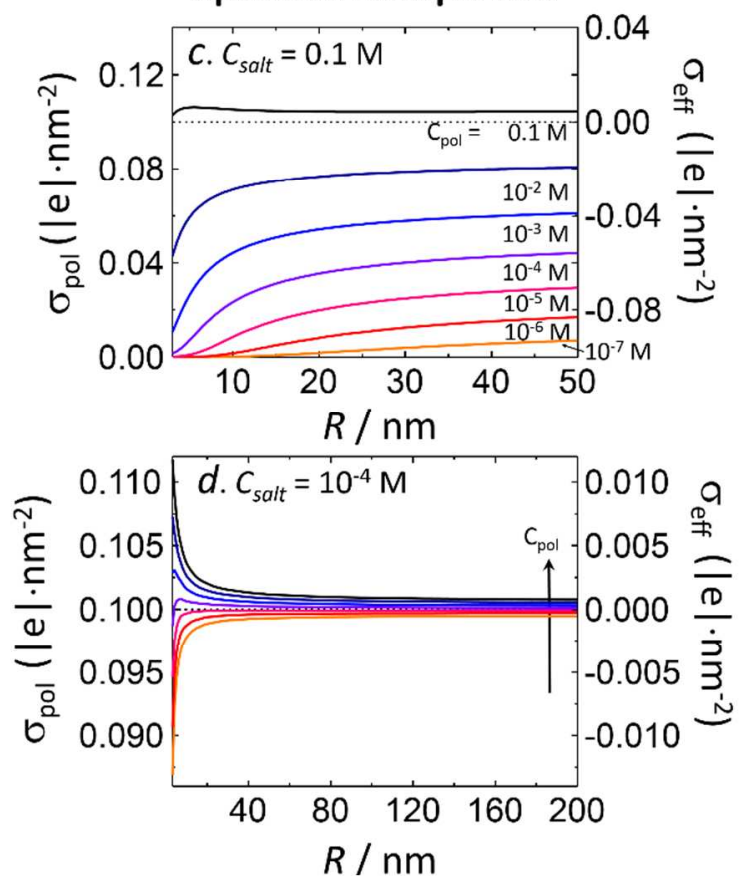

Figure 4. Same as Figure 1 but for polyelectrolyte adsorption on positively charged cylindrical nanorods (panels a and $\mathrm{b}$ ) or spherical nanoparticles (panels $\mathrm{c}$ and $\mathrm{d}$ ) of radius $R$. The bulk concentrations of the polyelectrolyte $\left(C_{\text {pol }}\right)$ and salt $\left(C_{\text {salt }}\right)$ are indicated in the plots. 
3.3. Conditions that maximize the effect of curvature on the amount of adsorbed polyelectrolyte

In order to evaluate the potential experimental relevance of the effect of surface curvature on polyelectrolyte adsorption, we compared polyelectrolyte adsorption on highly curved cylindrical nanorods and nanochannels $(R=7.5 \mathrm{~nm})$. Figure 5 shows the results of these calculations for $C_{\mathrm{pol}}=10^{-2} \mathrm{M}$ (concentrated polyelectrolyte solution, large chemical potential of polyelectrolyte) and $C_{\mathrm{pol}}=10^{-6} \mathrm{M}$ (diluted polyelectrolyte solution, small chemical potential of polyelectrolyte). Panels $\mathrm{a}$ and $\mathrm{b}$ in Figure 5 show $\sigma_{\mathrm{pol}}$ for these two polyelectrolyte concentrations as color maps as a function of chain length $(N)$ and bulk salt concentration $\left(C_{\text {salt }}\right)$ (the corresponding plots for the channel are shown in the Supporting Information Figure S2). For $C_{\mathrm{pol}}=10^{-2} \mathrm{M}, \sigma_{\mathrm{pol}}$ increases with chain length and experiences a maximum as a function of salt concentration, in agreement with previous reports ${ }^{1,18,20-21,28-29}$ This maximum is due the dual role of ionic strength, which screens both electrostatic repulsions among polyelectrolyte segments and electrostatic attractions between the segments and the surface. In the condition of the maximum, the charge of the polyelectrolyte overcompensates the charge of the surface $\left(\sigma_{\mathrm{pol}}\right.$ $\left.>0.1|\mathrm{e}| \cdot \mathrm{nm}^{-2}\right)$. For large salt concentrations, the polyelectrolyte completely desorbs from the surface. The critical salt concentration for which desorption occurs increases for increasing chain length, in line with previous simulation results. ${ }^{18}$ Panels $\mathrm{c}$ and $\mathrm{d}$ in Figure 5 show the difference between the amount of polyelectrolyte adsorbed on the rod and that on the channel, $\sigma_{\text {pol }}^{\text {rod }}-\sigma_{\text {pol }}^{\text {channel }}$ . Panels e and f show the ratio between these two quantities (we actually plot the logarithm of the ratio, $\left.\log _{10}\left(\sigma_{\text {pol }}^{\text {rod }} / \sigma_{\text {pol }}^{\text {chanel }}\right)\right)$. For $C_{\text {pol }}=0.01 \mathrm{M}$ (panels c and e), we observe that in the region where charge overcompensation occurs, the amount of adsorbed polyelectrolyte on the rod is predicted to be larger than on the channel ( $\sigma_{\text {pol }}^{\text {rod }}>\sigma_{\text {pol }}^{\text {channel }}$, red regions in panels c, d, e and f). The maximum value of $\sigma_{\text {pol }}^{\text {rod }}-\sigma_{\text {pol }}^{\text {channel }}$ is $\sim 0.011|\mathrm{e}| \cdot \mathrm{nm}^{-2}$, which is one order of magnitude smaller than the surface charge density $\left(\sigma_{\text {surf }}=-0.1|\mathrm{e}| \cdot \mathrm{nm}^{-2}\right)$. On the other hand, in these conditions, the ratio $\sigma_{\text {pol }}^{\text {rod }} / \sigma_{\text {pol }}^{\text {channel }}$ is close to 1 (i.e. $\log _{10}\left(\sigma_{\text {pol }}^{\text {rod }} / \sigma_{\text {pol }}^{\text {channel }}\right)$ close to zero, see scale of figure 5e) indicating that large composition deviations with surface curvature are unlikely to occur in the overcompensation regime. For $C_{\mathrm{pol}}=10^{-6} \mathrm{M}$, there is a region where charge undercompensation 
occurs and $\sigma_{\text {pol }}^{\text {channel }}>\sigma_{\text {pol }}^{\text {rod }}$ (blue region in panels $\mathrm{c}$ and $\mathrm{d}$, green region in panels e and $\mathrm{f}$ ). The maximum absolute difference in this region is $\sim 0.03|\mathrm{e}| \cdot \mathrm{nm}^{2}$ but since the amount of adsorbed polyelectrolyte is small, the relative ratio $\sigma_{p o l}^{\text {rod }} / \sigma_{\text {pol }}^{\text {channel }}$ can be significantly smaller than one ( $\log _{10}\left(\sigma_{\text {pol }}^{\text {rod }} / \sigma_{\text {pol }}^{\text {channel }}\right)<0$, see scale in figure $\left.5 f\right)$. In other words, large composition changes with surface curvature can occur in the charge undercompensation scenario, but at the cost of having a small polyelectrolyte coverage. We have also studied the effect of doubling the native surface charge, $\sigma_{\text {surf, }}$ which roughly doubles the absolute value of $\sigma_{p o l}^{\text {rod }}-\sigma_{\text {pol }}^{\text {channel }}$, but have little qualitative effect on the relative ratios $\sigma_{\text {pol }}^{\text {rod }} / \sigma_{\text {pol }}^{\text {chanel }}$ (see Figure 6)
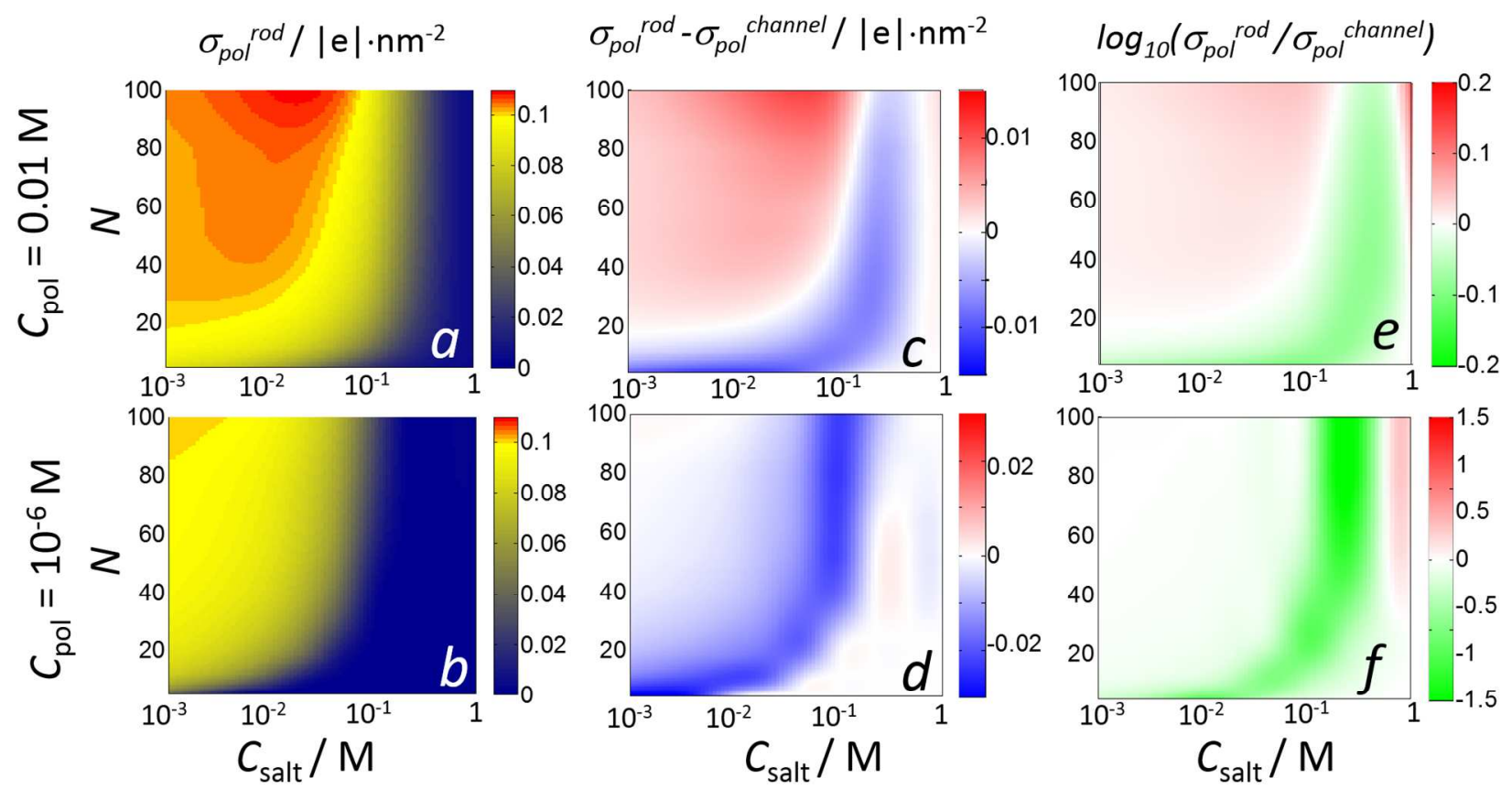

Figure 5: a, b) Color maps of the charge density of polyelectrolytes adsorbed on the surface of a cylindrical $\operatorname{rod}(R=7.5 \mathrm{~nm})$ as a function of bulk salt concentration $\left(C_{\text {salt }}\right)$ and chain length $(N)$ for $C_{\mathrm{pol}}=0.01 \mathrm{M}$ (panel a) and $C_{\mathrm{pol}}=10^{-6} \mathrm{M}$ (panel b). Note that $\sigma>0.1|\mathrm{e}| \cdot \mathrm{nm}^{-2}$ (red regions in the plots) indicates charge overcompensation. c, d) Color maps of the difference of the charge density of polyelectrolytes adsorbed on a rod $\left(\sigma_{\mathrm{pol}}{ }^{\mathrm{rod}}\right)$ and that of polyelectrolytes adsorbed on a cylindrical nanochannel $\left(\sigma_{\text {pol }}^{\text {channel }}\right)$ of the same radius and in the same conditions. e, f) Color 
maps of the logarithm of the ratio of the charge density of polyelectrolytes adsorbed on a rod to that of polyelectrolytes adsorbed on a cylindrical nanochannel. Note that in this scale, a value of 1 indicates $\sigma_{\mathrm{pol}}{ }^{\mathrm{rod}}=10 \cdot \sigma_{\mathrm{pol}}{ }^{\text {channel }}$ and a value of -1 indicates $\sigma_{\mathrm{pol}}^{\mathrm{rod}}=\sigma_{\mathrm{pol}}{ }^{\text {channel }} / 10$. The calculations correspond to a radius of curvature $R=7.5 \mathrm{~nm}$ and a surface charge of the substrate, $\sigma_{\text {surf }}=-0.1$ $|\mathrm{e}| \cdot \mathrm{nm}^{-2}$.
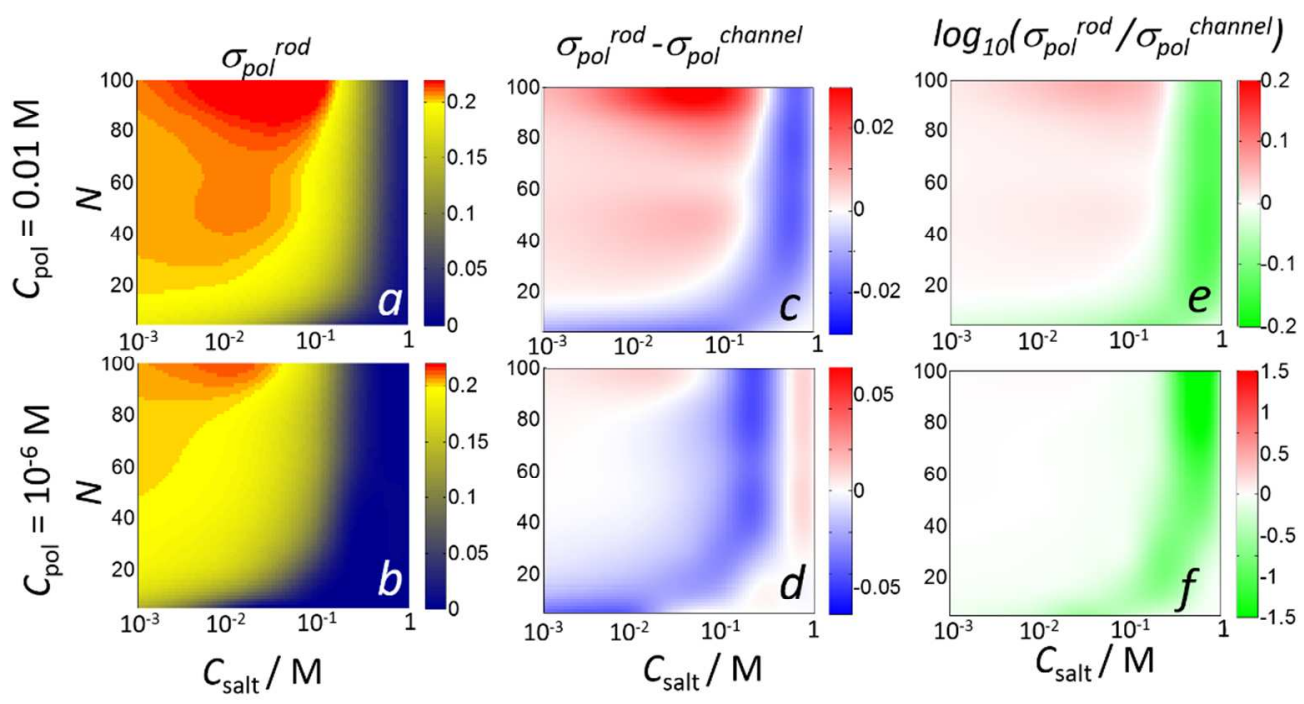

Figure 6. Same as Figure 5 for $\sigma_{\text {surf }}=-0.2|\mathrm{e}| \cdot \mathrm{nm}^{-2}$.

\section{Conclusions}

We reported on the mechanisms of modulation of polyelectrolyte adsorption by surface curvature. We show that increasing the curvature of the surface can lead to polyelectrolyte adsorption or desorption depending on the type of curvature (convex or concave) and whether the initial charge of the surface is over- or undercompensated by the polyelectrolyte. It is important to mention that our theoretical method have approximations that may affect our quantitative results; however, we believe that our explanation of the effect of surface curvature on polyelectrolyte adsorption is a general result that will qualitatively hold independently of these approximations. Regarding the approximations, our theory is mean-field, but the fact that we explicitly consider the conformations, shape and distribution of volume and charge of the 
polyelectrolyte (see derivation of the theory in methods section) introduces correlations into the distribution of the charges of the polyelectrolyte. These correlations allow our theory to capture some key aspects related to polyelectrolyte adsorption, such as the possibility of charge overcompensation, the non-monotonic effect of ionic strength on the amount of adsorbed polyelectrolyte (in the absence of additional non-electrostatic interactions) and the increase of the critical ionic strength for desorption with the chain length of the polyelectrolyte. Interestingly, the non-monotonic effect of ionic strength on the amount of adsorbed polyelectrolyte was previously observed in simulations ${ }^{18}$ and experiments, ${ }^{1,28-29}$ but it is not predicted by many other mean-field approaches. ${ }^{18,30-31}$ Another approximation involved in the present work is neglecting the end effects for nanochannels and nanorods (i.e. considering infinitely long channels and rods). This approximation results from the fact that we only allow inhomogeneities in the direction normal to the surface. Neglecting end effect will be a good approximation for systems with very large aspect ratios (such as ion-track etched nanochannels in polymer membranes that have aspect ratios of 100-1000 ${ }^{32}$ ). On the other hand, systems with small aspect ratios will require considering inhomogeneities in two or more coordinates, ${ }^{33-34}$ and will be addressed in future work.

We show that the differences in the amount of adsorbed polyelectrolytes between surfaces of different type of curvature are in general $<30 \%$ of the initial surface charge ( $\left.\sigma_{\text {surf }}\right)$. We thus believe that the effect of curvature on polyelectrolyte adsorption will be not be critical in most applications involving nanoobjects with only one type and one radius of curvature (e.g. spherical nanoparticles or cylindrical nanochannels). However, our results gain importance when different types or radii of curvature coexist within the same system, such as in polyelectrolyte adsorption on short nanorods (which have spherical and cylindrical curvature in the same particle) $)^{3}$ or conical nanochannels (where the radii of curvature at the tip and base can be very different). ${ }^{2}$ In such cases, our results can help to identify the conditions to reduce the inhomogeneity of the polyelectrolyte coverage on regions of different curvature, or even more interesting, to introduce patches of different polyelectrolyte composition by taking advantage of local curvature. 


\section{ASSOCIATED CONTENT}

Supporting Information. Numerical implementation, molecular model, effect of chain length and salt concentration on the amount of adsorbed polyelectrolyte on nanorods and nanochannels, table summarizing the symbols used in the theory.

\section{ACKNOWLEDGMENT}

MT and OA are fellows of CONICET. MT acknowledges financial support from Agencia Nacional de Promoción Científica y Tecnológica (ANPCyT) (PICT-0099-2015, PICT 01542016), IS thanks a grant from the NSF, CBET-1264696 and OA acknowledges support from CONICET (PIP0370) and ANPCyT (PICT-2013-0905, PICT-2016-1680)

\section{References}

1. Decher, G.; Schlenoff, B. J. Multilayer Thin Films; Wiley-VCH: Weinheim, 2003.

2. Ali, M.; Yameen, B.; Cervera, J.; Ramirez, P.; Neumann, R.; Ensinger, W.; Knoll, W.; Azzaroni, O. Layer-by-Layer Assembly of Polyelectrolytes Into Ionic Current Rectifying SolidState Nanopores: Insights from Theory and Experiment. J. Am. Chem. Soc. 2010, 132, 83388348.

3. Gole, A.; Murphy, C. J. Polyelectrolyte-Coated Gold Nanorods: Synthesis, Characterization and Immobilization. Chem. Mater. 2005, 17, 1325-1330.

4. Tagliazucchi, M.; Calvo, E. J.; Szleifer, I. Molecular Theory of Chemically Modified Electrodes by Redox Polyelectrolytes under Equilibrium Conditions: Comparison with Experiment. J. Phys. Chem. C. 2008, 112, 458-471.

5. Tagliazucchi, M.; Calvo, E. J.; Szleifer, I. Redox and Acid-Base Coupling in Ultrathin Polyelectrolyte Films. Langmuir 2008, 24, 2869-77.

6. Scodeller, P.; Flexer, V.; Szamocki, R.; Calvo, E. J.; Tognalli, N.; Troiani, H.; Fainstein, A. Wired-Enzyme Core-Shell Au Nanoparticle Biosensor. J. Am. Chem. Soc. 2008, 130, 1269012697.

7. Brunsen, A.; Calvo, A.; Williams, F. J.; Soler-Illia, G. J. A. A.; Azzaroni, O. Manipulation of Molecular Transport into Mesoporous Silica Thin Films by the Infiltration of Polyelectrolytes. Langmuir 2011, 27, 4328-4333.

8. Boroudjerdi, H.; Naji, A.; Naji, A.; Netz, R. Global Analysis of the Ground-State Wrapping Conformation of a Charged Polymer on an Oppositely Charged Nano-Sphere. European Physical Journal E 2014, 37, 21.

9. Shojaei, H.; Muthukumar, M. Adsorption and Encapsulation of Flexible Polyelectrolytes in Charged Spherical Vesicles. J. Chem. Phys. 2017, 146, 244901. 
10. Wang, J.; Muthukumar, M. Encapsulation of a Polyelectrolyte Chain by an Oppositely Charged Spherical Surface. J. Chem. Phys. 2011, 135, 194901.

11. Mella, M.; Izzo, L. Modulation of Ionization and Structural Properties of Weak Polyelectrolytes Due to 1d, 2d, and 3d Confinement. J. Polym. Sci., Part B: Polym. Phys. 2017, 55, 1088-1102.

12. Vázquez-Montejo, P.; McDargh, Z.; Deserno, M.; Guven, J. Cylindrical Confinement of Semiflexible Polymers. Phys. Rev. E 2015, 91, 063203.

13. de Carvalho, S. J.; Metzler, R.; Cherstvy, A. G. Inverted Critical Adsorption of Polyelectrolytes in Confinement. Soft Matter 2015, 11, 4430-4443.

14. Messina, R.; Holm, C.; Kremer, K. Polyelectrolyte Multilayering on a Charged Sphere. Langmuir 2003, 19, 4473-4482.

15. Narambuena, C. F.; Beltramo, D. M.; Leiva, E. P. Polyelectrolyte Adsorption on a Charged Surface. A Study by Monte Carlo Simulations. Macromolecules 2007, 40, 7336-7342.

16. Netz, R. R.; Andelman, D. Neutral and Charged Polymers at Interfaces. Phys. Rep. 2003, 380, 1-95.

17. Dobrynin, A. V.; Rubinstein, M. Theory of Polyelectrolytes in Solutions and at Surfaces. Progress in Polymer Science (Oxford) 2005, 30, 1049-1118.

18. Forsman, J., Polyelectrolyte Adsorption: Electrostatic Mechanisms and Nonmonotonic Responses to Salt Addition. Langmuir 2012, 28, 5138-5150.

19. Von Goeler, F.; Muthukumar, M. Adsorption of Polyelectrolytes onto Curved Surfaces. J. Chem. Phys. 1994, 100, 7796-7803.

20. Borukhov, I.; Andelman, D.; Orland, H. Scaling Laws of Polyelectrolyte Adsorption. Macromolecules 1998, 31, 1665-1671.

21. Cheng, H.; Olvera De La Cruz, M. Rod-Like Polyelectrolyte Adsorption Onto Charged Surfaces in Monovalent and Divalent Salt Solutions. J. Polym. Sci., Part B: Polym. Phys. 2004, 42, 3642-3653.

22. Cerda, J. J.; Qiao, B.; Holm, C. Understanding Polyelectrolyte Multilayers: An Open Challenge for Simulations. Soft Matter 2009, 5, 4412-4425.

23. Tagliazucchi, M.; Szleifer, I. Stimuli-Responsive Polymers Grafted to Nanopores and Other Nano-Curved Surfaces: Structure, Chemical Equilibrium and Transport. Soft Matter 2012, 8, 7292-7305.

24. Nap, R.; Gong, P.; Szleifer, I. Weak Polyelectrolytes Tethered to Surfaces: Effect of Geometry, Acid-Base Equilibrium and Electrical Permittivity. J. Polym. Sci., Part B: Polym. Phys. 2006, 44, 2638-2662.

25. Tagliazucchi, M.; Szleifer, I. How Does Confinement Change Ligand-Receptor Binding Equilibrium? Protein Binding in Nanopores and Nanochannels. J. Am. Chem. Soc. 2015, 137, 12539-12551.

26. Solveyra, E. G.; Tagliazucchi, M.; Szleifer, I. Anisotropic Surface Functionalization of $\mathrm{Au}$ Nanorods Driven by Molecular Architecture and Curvature Effects. Faraday Discuss. 2016, 191, 351-372.

27. Tagliazucchi, M.; Azzaroni, O.; Szleifer, I. Responsive Polymers End-Tethered in SolidState Nanochannels: When Nanoconfinement Really Matters. J. Am. Chem. Soc. 2010, 132, 12404-12411.

28. Liufu, S.-C.; Xiao, H.-N.; Li, Y.-P. Adsorption of Cationic Polyelectrolyte at the Solid/Liquid Interface and Dispersion of Nanosized Silica in Water. J. Colloid Interface Sci. 2005, 285, 33-40. 
29. Xie, F.; Nylander, T.; Piculell, L.; Utsel, S.; Wågberg, L.; Åkesson, T.; Forsman, J. Polyelectrolyte Adsorption on Solid Surfaces: Theoretical Predictions and Experimental Measurements. Langmuir 2013, 29, 12421-12431.

30. Van de Steeg, H. G. M.; Cohen Stuart, M. A.; De Keizer, A.; Bijsterbosch, B. H. Polyelectrolyte Adsorption: A Subtle Balance of Forces. Langmuir 1992, 8, 2538-2546.

31. Shafir, A.; Andelman, D.; Netz, R. R. Adsorption and Depletion of Polyelectrolytes from Charged Surfaces. J. Chem. Phys. 2003, 119, 2355-2362.

32. Tagliazucchi, M.; Szleifer, I., Chemically Modified Nanopores and Nanochannels; William Andrew, 2016.

33. Tagliazucchi, M.; Rabin, Y.; Szleifer, I. Ion Transport and Molecular Organization Are Coupled in Polyelectrolyte Modified Nanopores. J. Am. Chem. Soc. 2011, 133, 17753-17763.

34. Peleg, O.; Tagliazucchi, M.; Kroeger, M.; Rabin, Y.; Szleifer, I. Morphology Control of Hairy Nanopores. ACS Nano 2011, 5, 4737-4747. 


\section{TOC Graphic}

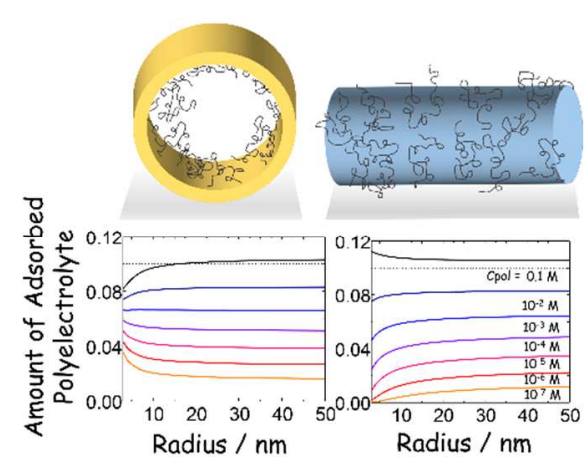

